\title{
Technology of Thin Film Fabrication on Porous Metal Oxide Substrates
}

\author{
Yu.I. Shtern*, M.Yu. Shtern, R.E. Mironov, A.A. Sherchenkov and M.S.Rogachev \\ National Research University of Electronic Technology - MIET, \\ Bld. 1, Shokin Square, Zelenograd, Moscow, Russia, 124498
}

\begin{abstract}
One of the main issues of the device technology on metal-dielectric (MD) substrates is poor adhesion of thin metallic films, which are used for interconnections in electrical circuits. Films are formed by vacuum deposition on a porous dielectric layer of substrates. The presence of pores sufficiently complicates the cleaning of the substrate surface, which significantly decreases the adhesion of the film deposited on it. Technology of metal films with high adhesion, fabricated by ion-plasma method is proposed in this work. Films were deposited on MD substrates produced by electrochemical oxidation of aluminum alloys. The main operations of the technology are the following. Removal of residual electrolyte after the oxidation is carried out by rinsing of substrates initially under running hot water, and then in deionized water, followed by drying in a flow of heated nitrogen. Annealing of substrates under the pressure of $10^{-3} \mathrm{~Pa}$ and temperature of $550-570 \mathrm{~K}$ for $20 \mathrm{~min}$ is carried on in the vacuum chamber before the deposition of metal films. Copper is used as the main material of the interconnection films. Adhesion sublayer is fabricated on the basis of chromium or vanadium, which have high enthalpy of the oxide formation. Measurements showed that the copper films with the thickness of $1.5 \mu \mathrm{m}$, deposited on the vanadium sublayer with the thickness of $0.12 \mu \mathrm{m}$, which is comparable with the roughness of oxide layer, have adhesion of $25 \mathrm{~N} / \mathrm{mm}^{2}$ at the temperature of $520 \mathrm{~K}$. Investigation of adhesion was carried on by the method of direct tear off with the error of up to $10 \%$.
\end{abstract}

DOI: 10.12693/APhysPolA.129.776

PACS/topics: $68.35 . \mathrm{Np}$

\section{Introduction}

Metal-dielectric structures are frequently used as substrates in electron devices. Best quality have metaldielectric substrates produced by electrochemical oxidation of aluminum alloys $[1,2]$. These substrates have high dielectric properties together with high mechanical strength and thermal conductivity. It is necessary to note that porous oxide layers are perspective materials for the fabrication of electron devices with the use of achievements of nanotechnology.

One of the main problems of the technology of devices on the MD substrates is low adhesion of metal thin films, which are used for interconnections in electrical circuits. Films are formed by vacuum deposition on a porous dielectric layer of substrates. The presence of pores sufficiently complicates the cleaning of the substrate surface, which significantly decreases adhesion of the film deposited on it. The density of pores fabricated in dissolving the oxide layer electrolyte can be as high as $10^{9}$ $10^{10}$ pores $/ \mathrm{cm}^{2}$, and can reach $36 \%$ of the volume of the oxide layer. Diameters of the pores are usually varied in the range of $10-100 \mathrm{~nm}$.

Influence on the adhesion of such factors as methods of surface treatment of substrates, regimes of substrate annealing in vacuum, metal properties, thicknesses of adhesion sublayer and main conducting layer, and regimes of metal film deposition were investigated in this work

*corresponding author; e-mail: hptt@miee.ru during the optimization of the technology of metal film fabrication on the MD substrates with the aim of obtaining of maximum adhesion.

The value of adhesion cannot be accurately calculated. So, experimental investigation of adhesion strength, which is necessary for estimation of adhesion quality, is quite actual now. Adhesion strength qualitatively characterizes true adhesion [3]. Investigation of adhesion strength was carried on by the method of direct tear off with the error of up to $10 \%$.

\section{Preparation of substrate surface before the metallization}

Condition of the substrate surface is the decisive factor determining adhesion of the films [4]. Preliminary cleaning of the substrate surface and its control plays crucial role in the ensuring of good adhesion of the films. Cleaning of the surface from adsorbed gases and dopants is connected with breaking of the bonds between the impurity molecules, and between impurity molecules and the substrate. MD substrates are active adsorbents due to the high specific surface area. The most dangerous are organic contaminations, which can decompose during the deposition of thin films due to the heating of substrates and due to electron and ion bombardment. Emitted gaseous products $\left(\mathrm{H}_{2} \mathrm{O}, \mathrm{CO}_{2}, \mathrm{CO}, \mathrm{O}_{2}\right.$, etc. $)$ negatively influence the formation of thin films, decreasing their adhesion. Most effective method of degasing porous substrates is thermal treatment in vacuum. The term degassing means gas release due to desorption and diffusion. The rate of impurity desorption has exponential temperature dependence, and so sharply increases with heating. 
Low pressure in a vacuum chamber sufficiently promotes degasing of substrates.

In the developed technology removal of residual electrolyte after the oxidation is carried out by rinsing of substrates initially under running hot water, and then in deionized water, followed by drying in a flow of heated nitrogen. Annealing of substrates under the pressure of $10^{-3} \mathrm{~Pa}$ was carried on in the vacuum chamber before the deposition of metal films. This operation sufficiently helps cleaning of the substrate and increases adhesion of the deposited films. Dependences of the film adhesion strength on time of substrate annealing in vacuum are presented in Fig. 1.

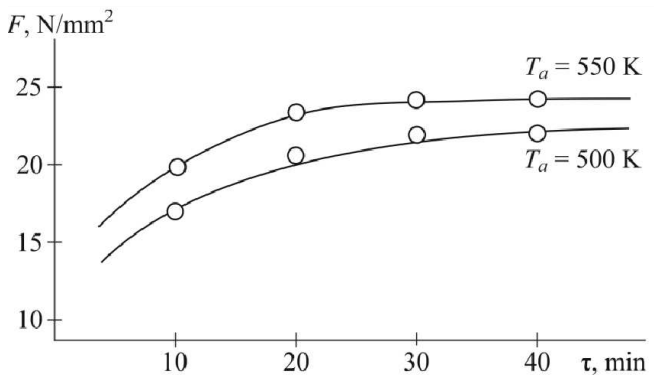

Fig. 1. Dependences of the film adhesion strength $F$ on the time of substrate annealing in vacuum.

The optimal temperature of annealing in vacuum is $550-570 \mathrm{~K}$, while annealing time is $20 \mathrm{~min}$, which provide maximum adhesion of metal films.

Investigation of adhesion strength showed that chemical cleaning of the porous substrate surface negatively influences the adhesion of thin films. This result can be explained by the difficulty of removing the solvent from the porous substrate surface. Negative phenomena, which accompany treatment of substrates by different solvents, are discussed in [5].

\section{Preparation and investigation of metal thin films on MD substrates}

In the developed technology copper is used as the main material of the interconnection films, which are deposited by ion-plasma method. It is known that metals with high conductivity applied for the fabrication of interconnections usually possess low adhesion properties. That is why additional adhesion sublayers are used in thin film technology. The use of a sublayer sufficiently increases adhesion, if chemical bonds between the film and substrate are formed on the initial stage of deposition. For the substrates on the basis of metal oxide, the chemisorption occurs during the deposition of oxides and metals, easily interacting with oxygen. In this connection for the formation of adhesion sublayer are used metals with sufficient enthalpy of formation of oxide, such as $\mathrm{Cr}, \mathrm{Al}$, Ti, Fe, V.

We have used the adhesion sublayer of chromium or vanadium. Investigations showed that the interconnections formed with vanadium sublayer have higher adhesion strength (by 10-15\%). This result can be explained by the higher enthalpy of formation of oxide per atom, than that of chromium. In addition, as will be shown below, thermal component of the internal stress for chromium film is lower than that for vanadium layer.

Relatively high roughness of MD substrates increases the contact area of deposited films, which promotes the increase of the adhesion strength. However, such surface morphology requires specific conditions of adhesion sublayer formation. In particular, the critical thickness of the sublayer increases sufficiently. We have deposited copper films (thickness of $1.5 \times 10^{-6} \mathrm{~m}$ ) with vanadium adhesion sublayers with different thicknesses (from $150 \times 10^{-10} \mathrm{~m}$ to $1400 \times 10^{-10} \mathrm{~m}$ ). For each vanadium sublayer thickness we have used two substrates, and deposited metal films at temperatures of 420, 470, and $520 \mathrm{~K}$. Results of investigation of adhesion strength (Fig. 2) allowed us to determine the optimal thickness of vanadium adhesion sublayer, and temperature of film deposition.

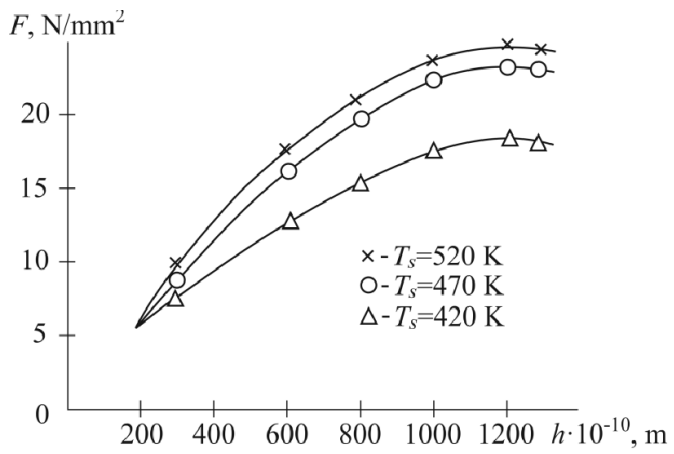

Fig. 2. Dependences of adhesion strength on the thickness $h$ of vanadium sublayer at different deposition temperatures.

Vanadium and copper were deposited on the MD substrates with roughness $\sim 1100 \times 10^{-10} \mathrm{~m}$. As can be seen from Fig. 2, the maximum adhesion strength is achieved for the vanadium thickness of $(1000-1200) \times 10^{-10} \mathrm{~m}$, which is comparable with the roughness of substrate surface. Increase of the thickness of vanadium film above $0.12 \mu \mathrm{m}$ is useless because it does not increase the adhesion strength.

Annealing in vacuum before the deposition of metal film, and high temperature of substrate just before the deposition cleans the surface, which promote at the initial stage of deposition the formation of oxide bonds between metal layer and the substrate. Probability of chemical bond formation increases for the metals possessing high energy of oxide formation. After the formation of metaloxide bonds on the substrate surface, adhesion of the film will be determined by metal bonds. Adhesion of the metal films due to the formation of oxide bonds is explained in [3]. Formation of chemical bonds between the adsorbate and atoms, or group of atoms on the surface of the adsorbate due to the existence of dangling bonds is discussed for insulators and semiconductors in [4]. 
As was mentioned above, one of the main factors, which influences the formation of thin films, is the substrate temperature. At the first stage of the process, the annealing of substrates at temperatures of $550-570 \mathrm{~K}$ in vacuum is carried out. After that the substrate is cooled to the temperature of $500 \mathrm{~K}$. Then film deposition begins, and substrate temperature increases to the temperature of $520 \mathrm{~K}$. Evolution of the substrate temperature during the process of metal film formation is presented in Fig. 3. It is important to note that substrate temperature during the metal film deposition must not exceed vacuum annealing temperature.

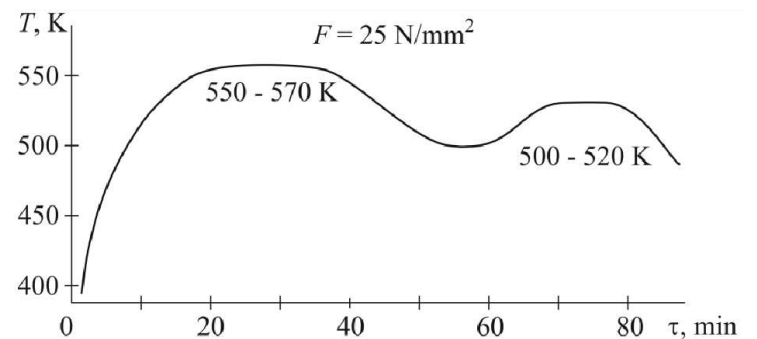

Fig. 3. Variation of the substrate temperature during the process of metal film formation.

Investigations have shown that technology allows fabrication of metal films with the adhesion strength of up to $25 \mathrm{~N} / \mathrm{mm}^{2}$, with the variation of adhesion strength on the substrate not exceeding $1 \mathrm{~N} / \mathrm{mm}^{2}$.

High substrate temperature during the metal film deposition $(520 \mathrm{~K})$ promotes formation of chemical bonds between the adsorbent and adsorbate. Mobility of adsorbed atoms increases with the increase of substrate temperature, which helps the uniform deposition of metal film on the rough surface of the substrate, thus maximizing the area of film-to-substrate contact. This fact is very important for the application of MD substrates on the basis of oxidized aluminum with the high surface roughness of $0.12 \mu \mathrm{m}$. It is necessary to note that high mobility of atoms orders the film structure, shifting it closer to the equilibrium [5]. This decreases stresses in the film, which is crucial for this temperature range.

The following types of stresses appear in the fabricated films: (i) intrinsic, connected with the film structure and existing defects, and (ii) thermal, which is due to the difference of the coefficients of linear thermal expansion (CLTE) of the film and substrate. Stresses in the film may be of the order of adhesion strength. Stresses in the film depend on the surface roughness and film thickness [3].

Thermal component of film stresses for copper, chromium and vanadium layers were calculated using the equation

$$
\varepsilon_{\mathrm{T}}=\frac{E}{1-\nu}\left(\alpha_{\mathrm{F}}-\alpha_{\mathrm{S}}\right)\left(T_{\mathrm{S}}-T_{0}\right)
$$

where $\nu$ is Poisson coefficient for the film material; $E$ is Young modulus for the film material, $\mathrm{Pa} ; \alpha_{F}, \alpha_{S}$ are
CLTE of the film and substrate, $\mathrm{K}^{-1} ; T_{0}$ is operation temperature of the substrate, $\mathrm{K} ; T_{S}$ is substrate temperature during deposition, K.

CLTE for the oxidized aluminum was assumed to be equal to $17 \times 10^{-6} \mathrm{~K}^{-1}$. Results of the calculations show that the difference of CLTE for substrate and films causes compression stress in the layer. It was found that chromium films have the largest value of thermal component of film stress $\left(\varepsilon_{\mathrm{T}, \mathrm{Cu}}=-14 \times 10^{6} \mathrm{~Pa} ; \varepsilon_{\mathrm{T}, \mathrm{V}}=\right.$ $\left.-380 \times 10^{6} \mathrm{~Pa} ; \varepsilon_{\mathrm{T}, \mathrm{Cr}}=-1140 \times 10^{6} \mathrm{~Pa}\right)$. Adhesion strength for the vanadium sublayer is higher (by 10-15\%) in comparison with that of chromium sublayer.

\section{Conclusions}

One of the main issues of the device technology on MD substrates is poor adhesion of thin metallic films, which are used for interconnections in electrical circuits. Films are formed by vacuum deposition on a porous dielectric layer of substrates.

Technology of metal films with high adhesion fabricated by ion-plasma method is proposed. The main operations of the technology are the following. Removal of residual electrolyte after the oxidation is carried out by rinsing of substrates initially under running hot water, and then in deionized water, followed by drying in a flow of heated nitrogen. Annealing of substrates under the pressure of $10^{-3} \mathrm{~Pa}$ and temperature of 550$570 \mathrm{~K}$ for $20 \mathrm{~min}$ is carried on in the vacuum chamber before the deposition of metal films. Copper is used as the main material for the interconnection films. Adhesion sublayer is fabricated on the basis of chromium or vanadium, which have high enthalpy of the oxide formation. Measurements showed that the copper films with the thickness of $1.5 \mu \mathrm{m}$, deposited on the vanadium sublayer with the thickness of $0.12 \mu \mathrm{m}$, which is comparable with the roughness of oxide layer, have the adhesion of $25 \mathrm{~N} / \mathrm{mm}^{2}$ at the temperature of $520 \mathrm{~K}$. Investigation of adhesion was carried on by the method of direct tear off, with the error of up to $10 \%$.

\section{Acknowledgments}

This work was supported by the Ministry of Education and Science of Russian Federation project № 14.578.21.0038 (RFMEFI57814X0038).

\section{References}

[1] Yu.I. Shtern, Russ. J. Appl. Chem. 81, 585 (2008).

[2] S. Altuntas, F. Buyukserin, Appl. Surf. Sci. 318, 290 (2014).

[3] K.L. Mittal, Tanweer Ahsan. Adhesion in Microelectronics, Wiley-Scrivener, 2014, p. 368.

[4] S.R. Morrison, The Chemical Physics of Surfaces, Springer US, 1990, p. 438.

[5] A.J. Kinlok, Adhesion and adhesives, 1987, p. 441. 